\title{
STUDIES ON MECHANISM OF SAND REMOVAL FROM CRUDE OIL
}

Guo Jun ${ }^{1}$, Gong Dao-tong ${ }^{1}$, Zhang jun ${ }^{1}$, Wang Li-yang ${ }^{1}$, Zheng Zhi-chu ${ }^{1}$ Li Ke-ming ${ }^{2}$

1. Institute of Mechanics, Chinese Academy of Sciences , Beijing 100080, China

2. China Aviation Oil Supply Co., Ltd. HeBei Branch, Shijiazhuang, China

\begin{abstract}
Numerical simulation and experimental research on sand removal was performed. The sedimentation mechanism of sand particles of various diameters was investigated under different oil/water ratios and oil viscosities. Moreover, commercial CFD software FLUENT was applied to simulate numerically gravitational sedimenttation separation. Through the comparison between simulation and experimental results, a kind of high-efficient sand-removal method was put forward. The work was completed jointly by the Institute of Mechanics, CAS and China National Offshore Oil Corporation during the "tenth-five-year" period.
\end{abstract}

KEY WORDS: centrifugal separation, oil/sand separation, particle sedimentation

\section{Introduction}

At present, sand is a large threat to the daily operation in oil fields, and must be removed with the oil/gas/sand separation. In gathering/transportation system on ground, several methods, including filtering, gravitation, rotation, heating and chemical treatment, have been applied for the removal of sand. As a kind of direct and traditional separation technique, gravitational sedimentation is applied most widely in oil fields. However, this technique cannot meet the requirements of removing small-diameter sand particles as the viscosity of crude oil increases. Centrifugal separation is another popular technique used for sand removal on ground, and the requirement of removing small-diameter sand particles was met in our cooperative research. Based on theoretical analyses and experimental study, a new set of centrifugal sand-removal equipment is developed.

\section{Numerical Simulation}

To realize the removal of sand in oil, the sedimentation mechanism of sand particles is a key in achieving high-efficiency oil/sand separation. The commercial CFD software FLUENT is applied to numerically simulate the motion of sand particles of various diameters within liquids of different viscosities, especially gravitational sedimentation of sand particles within highly viscous crude oil, where interaction between each phase, as well as the viscosity of oil, is considered.

\subsection{Fundamental equations \\ 2.1.1 Continuity equation}

The continuity equation for phase $\boldsymbol{q}$ is

$\frac{\partial}{\partial t}\left(\alpha_{q} \rho_{q}\right)+\nabla \cdot\left(\alpha_{q} \rho_{q} \vec{v}_{q}\right)=\dot{m}_{p q}$

\subsubsection{Momentum equation}

The momentum balance for phase $\boldsymbol{q}$ is

$\frac{\partial}{\partial t}\left(\alpha_{q} \rho_{q} \vec{v}_{q}\right)+\nabla \cdot\left(\alpha_{q} \rho_{q} \vec{v}_{q} \vec{v}_{q}\right)=$

$-\alpha_{q} \nabla p+\nabla \cdot \overline{\overline{\tau_{q}}}+\alpha_{q} \rho_{q} \vec{g}$

$+\alpha_{q} \rho_{q}\left(\vec{F}_{q}+\vec{F}_{l i f t, q}+\vec{F}_{V m, q}\right)$

$+\sum_{p=1}^{n}\left(K_{p q}\left(\vec{v}_{p}-\vec{v}_{q}\right)+\dot{m}_{p q} \vec{v}_{p q}\right)$

where $p$ is the pressure for all phases and $\vec{v}_{p q}$ is the interphase velocity, defined as follows. If $\dot{m}_{p q}>0$ (i.e., the mass of phase $p$ is being transferred to phase q), $\vec{v}_{p q}=\vec{v}_{p}$; if $\dot{m}_{p q}<0$ (i.e., the mass of phase $q$ is being transferred to phase $p$ ), $\vec{v}_{p q}=\vec{v}_{q}$. Herein, $K_{p q}$ is the interphase momentum exchange coefficient, and depends on friction, cohesion, etc.

The momentum equation for solid phase $S$ is

$$
\begin{aligned}
& \frac{\partial}{\partial t}\left(\alpha_{s} \rho_{s} \vec{v}_{s}\right)+\nabla \cdot\left(\alpha_{s} \rho_{s} \vec{v}_{s} \vec{v}_{s}\right)= \\
& -\alpha_{s} \nabla p-\nabla p_{s}+\nabla \cdot \bar{\tau}_{s}+\alpha_{s} \rho_{s} \vec{g} \\
& +\alpha_{s} \rho_{s}\left(\vec{F}_{s}+\vec{F}_{l i f t, s}+\vec{F}_{V m, s}\right)+ \\
& \sum_{l=1}^{n}\left(K_{l s}\left(\vec{v}_{l}-\vec{v}_{s}\right)+\dot{m}_{l s} \vec{v}_{l s}\right)
\end{aligned}
$$

The exchange coefficient $K_{s l}$ between oil and sand particles can be expressed as 


$$
K_{s l}=\frac{\alpha_{s} \rho_{s} C_{D} \operatorname{Re}_{s} \alpha_{l}}{24 v_{r, s}^{2} \tau_{s}}
$$

where the relaxation time $\tau_{s}$ of solid particles is defined as

$$
\tau_{s}=\frac{\rho_{s} d_{s}^{2}}{18 \mu_{l}}
$$

The drag coefficient was given by Dalla Valle ${ }^{[3]}$ :

$$
C_{D}=\left(0.63+\frac{4.8}{\sqrt{\operatorname{Re}_{s} / v_{r, s}}}\right)^{2}
$$

This model is based on the measurements of the terminal velocities of particles, with correlations that are a function of the volume fraction and relative Reynolds number:

$$
\operatorname{Re}_{s}=\frac{\rho_{l} d_{s}\left|\vec{v}_{s}-\vec{v}_{l}\right|}{\mu_{l}}
$$

The exchange coefficient among sand particles can be expressed as

$$
\begin{aligned}
& K_{l s}=\frac{3\left(1+e_{l s}\right)\left(\frac{\pi}{2}+C_{f r, l s} \frac{\pi^{2}}{8}\right)}{2 \pi\left(\rho_{l} d_{l}^{3}+\rho_{s} d_{s}^{3}\right)} \\
& \times \alpha_{s} \rho_{s} \alpha_{l} \rho_{l}\left(d_{l}+d_{s}\right)^{2} g_{o, l s}\left|\vec{v}_{l}-\vec{v}_{s}\right|
\end{aligned}
$$

$$
\text { where } C_{f r, l s}=0 \text { for solid particles. }
$$

\subsubsection{Turbulence model}

The gravitational sedimentation is relatively slow, and its velocity is less than $1.0 \times 10^{-2} \mathrm{~m} / \mathrm{s}$. Therefore, laminar model is chosen.

\subsubsection{Boundary and initial conditions}

According to the characteristics of selected model, the boundary conditions are set as solid wall, namely, the adhesion conditions are given, while both the velocity and turbulent intensity are zero. Uniform mixture within the flow field is initialized at $t=0$ and the velocity is also initialized to be zero.

\subsubsection{Numerical model}

Eulerian model of the Euler-Euler methods is applied to numerically simulate the separation of oil and sand particles, where the Syamlal-O'Brien ${ }^{[6]}$ model is chosen to describe the interaction between phases.

\section{Numerical Simulation}

\subsection{Numerical model}

Sand particles of various diameters were mixed with a certain oil/water-ratio liquid mixture in polymethyl methacrylate tank. The height of liquid mixture is $410 \mathrm{~mm}$ (see Fig. 1). The diameters of tank bottom in both the model and experiment are $1 \mathrm{~m}$ in order to compare the numerical results and experimental data. Due to symmetry of flow field within the tank, a section in the axial direction is taken in CFD calculation, and thus the three-dimensional simulation can be simplified to a two-dimensional one. In the model, the liquid mixture is supposed to be the continuous phase as it occupies most of the space. The volume ratio of oil/sand was taken to be 10 percent, a little more than the practical value in the experiments.

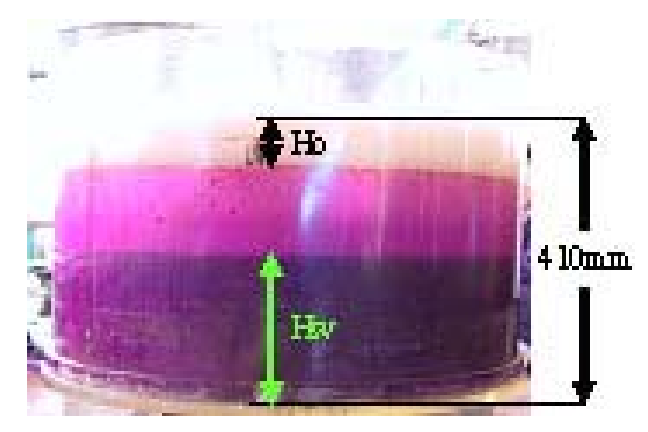

Fig. 1 Oil/Water-mixture tank

Physical properties for each phase in the model are shown in Table 1:

Table 1 Physical properties for each phase

\begin{tabular}{|c|c|c|}
\hline & Oil & Sand \\
\hline Density $\left(\mathrm{kg} / \mathrm{m}^{3}\right)$ & 836 & 2580 \\
\hline Viscosity $(\mathrm{mpa} \cdot \mathrm{s})$ & $31,100,300,800$ & \\
\hline
\end{tabular}

\subsection{Numerical algorithm}

The commercial CFD software FLUENT is used to conduct the numerical simulation, where the SIMPLE algorithm ${ }^{[7]}$ based on finite volume is applied. A P-V modified algorithm is adopted to discretize the equations.

\section{Results and Analyses}

\subsection{Oil/sand separation and conclusions}

The variation of concentration distribution with time during the sedimentation is shown in Fig 3 . Time needed for sand particles of different diameters inside oil with different viscosities is also given in Table 2 . As can be seen, it takes much longer time to sedimentate as the diameter of sand particles become 
smaller and the oil viscosity increases, and vice versa. It is very difficult for small-diameter sand particles to sedimentate in high-viscosity crude oil through gravitational sedimentation. The difference between oil/sand mixture and oil/water one is that the latter's transition layer is more uniform, which is advantageous in the separation. The smaller the diameter of sand particles is, the wider the boundary of sand particle accumulating layer will be.

Table 2 Time needed for gravitational sedimentation of oil/sand mixture

\begin{tabular}{|c|c|c|c|c|c|}
\hline \multirow{2}{*}{$\begin{array}{c}\text { Oil viscosity } \\
\mathrm{mPa} \cdot \mathrm{s}\end{array}$} & \multicolumn{5}{|c|}{ Sedimentation time of sand particles (s) } \\
\cline { 2 - 6 } & 20 eyes & 40 eyes & 60 eyes & 80 eyes & 100 eyes \\
\hline 31 & 33 & 130 & 310 & 811 & 1100 \\
\hline 100 & 105 & 400 & 1000 & 2600 & 3400 \\
\hline 300 & 310 & 1200 & 3150 & 7200 & 10800 \\
\hline 800 & 840 & 3200 & 8200 & 19200 & 26400 \\
\hline
\end{tabular}

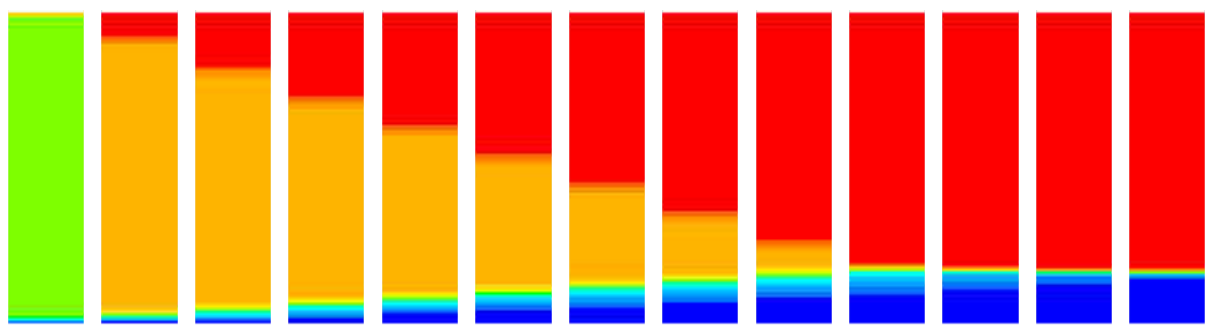

Fig. 3 Variation of sand particle concentration distribution with time

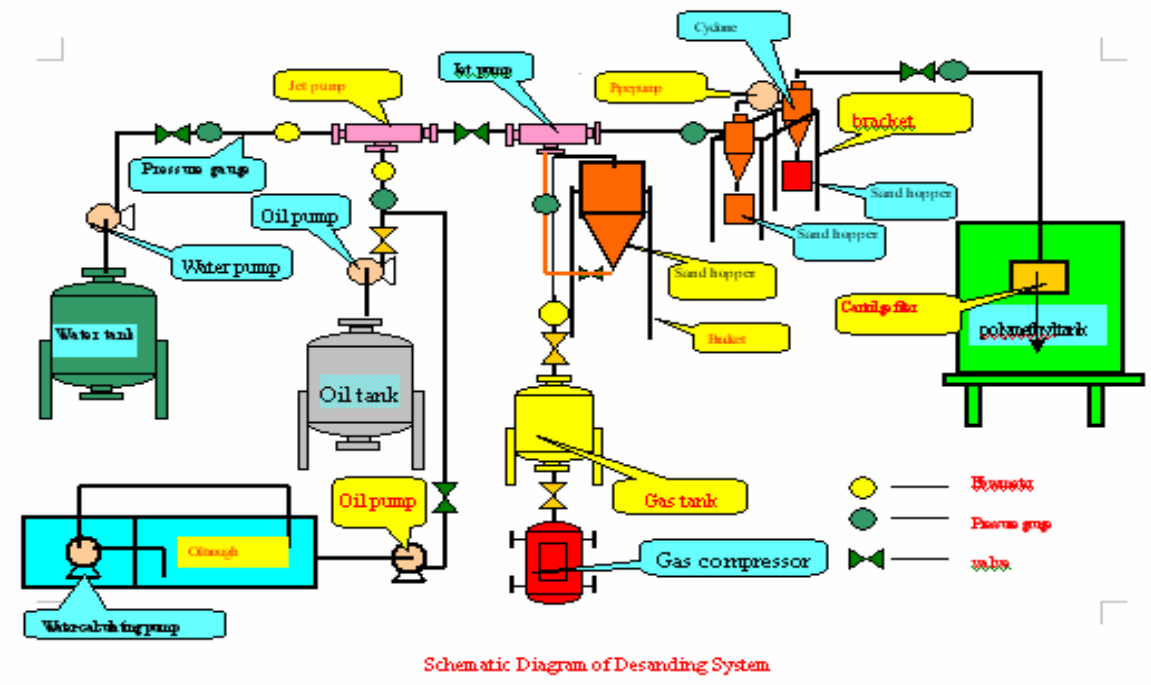

Fig. 4 Schematic diagram of desanding system

\subsection{Conclusions}

The following conclusions can be reached:

a. It takes more time to separate sand from oil as the oil viscosity increases and the sand particle diameters become smaller, and vice versa.

b. For small-diameter sand particles or in case of highly viscous oil, the separation efficiency of gravitational sedimentation is relatively low. Therefore, some other ways should be considered

\section{Experiments}

\subsection{Experimental system}

To achieve high-efficiency separation, some other ways should be applied besides gravitational sedimentation. It is a good idea to improve the desanding efficiency through centrifugal force. Therefore, a set of liquid/solid separation system was set up in the Institute of Mechanics, CAS, and its sketch is given in Fig. 4. 


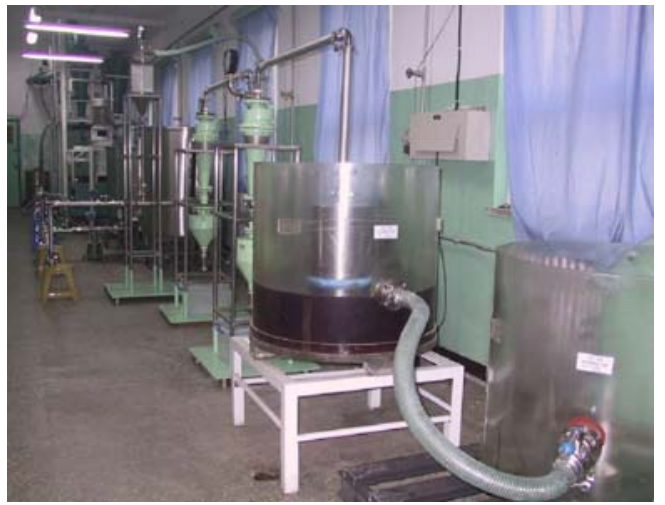

Fig. 5 Experimental system

Oil, water and sand particles are mixed using jet pump, which would be separated in two-stage cyclone separators to improve the efficiency. Three key equipments are available in the system: (I) Sand-adding device, including gas inlet pipe, water inlet pipe, sand-adding pipe, pressure gauge, valves and so on; (II) Catcher, consisting of first-stage filtering screen, second-stage screen and overflow external cylinder;
(III) Separator, essential part of liquid/solid separation, where oil and solid are separated based on the principle of rotational flow. The main characteristics of such a separator are its high processing capacity and compactness.

\subsection{Experimental results}

The flow media include LP14 oil, water and sand particles, and the related properties are given in Table 3 , and a set of typical data in Table 4 .

a. Rotational separation is very valuable in improving the efficiency of oil/sand separation, where centrifugal force is the key in determining the efficiency of small-diameter sand particles.

b. For various oil viscosities, the separation efficiency of sand particles with diameters more than 100 eyes can arrive at 95 percent.

Table 3 Experimental parameters

\begin{tabular}{|c|c|c|c|c|c|c|c|c|}
\hline $\begin{array}{c}\text { Transient } \\
\text { Oil } \\
\begin{array}{c}\text { Flux } \\
\left(\mathrm{m}^{3} / \mathrm{h}\right)\end{array}\end{array}$ & $\begin{array}{c}\text { Transient } \\
\text { Water } \\
\text { Flux } \\
\left(\mathrm{m}^{3} / \mathrm{h}\right)\end{array}$ & $\begin{array}{c}\text { Total } \\
\text { Flux } \\
\left(\mathrm{m}^{3} / \mathrm{h}\right)\end{array}$ & $\begin{array}{c}\text { Flowrate } \\
\text { Inside } \\
\text { Main } \\
\text { Pipe } \\
(\mathrm{m} / \mathrm{s})\end{array}$ & $\begin{array}{c}\text { Oil } \\
\text { Pressure } \\
(\mathrm{Mpa})\end{array}$ & $\begin{array}{c}\text { Water } \\
\text { Pressur } \\
(\mathrm{Mpa})\end{array}$ & $\begin{array}{c}\text { Air } \\
\text { Pressure } \\
(\mathrm{Mpa})\end{array}$ & $\begin{array}{c}\text { Pressure at } \\
\text { the inlet } \\
\text { of } \\
\text { First-stage } \\
\text { Cyclone } \\
(\mathrm{Mpa})\end{array}$ & $\begin{array}{c}\text { Pressure } \\
\text { at the } \\
\text { inlet of } \\
\text { Second } \\
\text {-stage } \\
\text { cyclone } \\
(\mathrm{Mpa})\end{array}$ \\
\hline 6.88 & 4.917 & 11.80 & 2.61 & 0.57 & 0.565 & 0.08 & 0.125 & 0.075 \\
\hline
\end{tabular}

Table 4 Parameters before and after separation

\begin{tabular}{|c|c|c|c|c|}
\hline $\begin{array}{c}\text { Particle Diameter } \\
\text { Mass of sand particles } \\
\text { before experiments(g) }\end{array}$ & $20-40$ eyes & $40-60$ eyes & $60-80$ eyes & $<80$ eyes \\
\cline { 2 - 5 } & 1758.5 & 241.5 & 100 & 0 \\
\hline $\begin{array}{c}\text { Separation Mass at the } \\
\text { first-stage cyclone (g) }\end{array}$ & 1503.1 & 320.8 & 72.9 & 23 \\
\hline $\begin{array}{c}\text { Separation Mass at the } \\
\text { second-stage cyclone } \\
\text { (g) }\end{array}$ & 0 & 10.5 & 72.3 & 39.5 \\
\hline Mass inside Catcher (g) & 0 & 0 & 0 & 16 \\
\hline Total Separation Mass & & \multicolumn{5}{|c|}{2058.1} \\
\hline Desanding Efficiency & \multicolumn{5}{|c|}{} \\
\hline
\end{tabular}



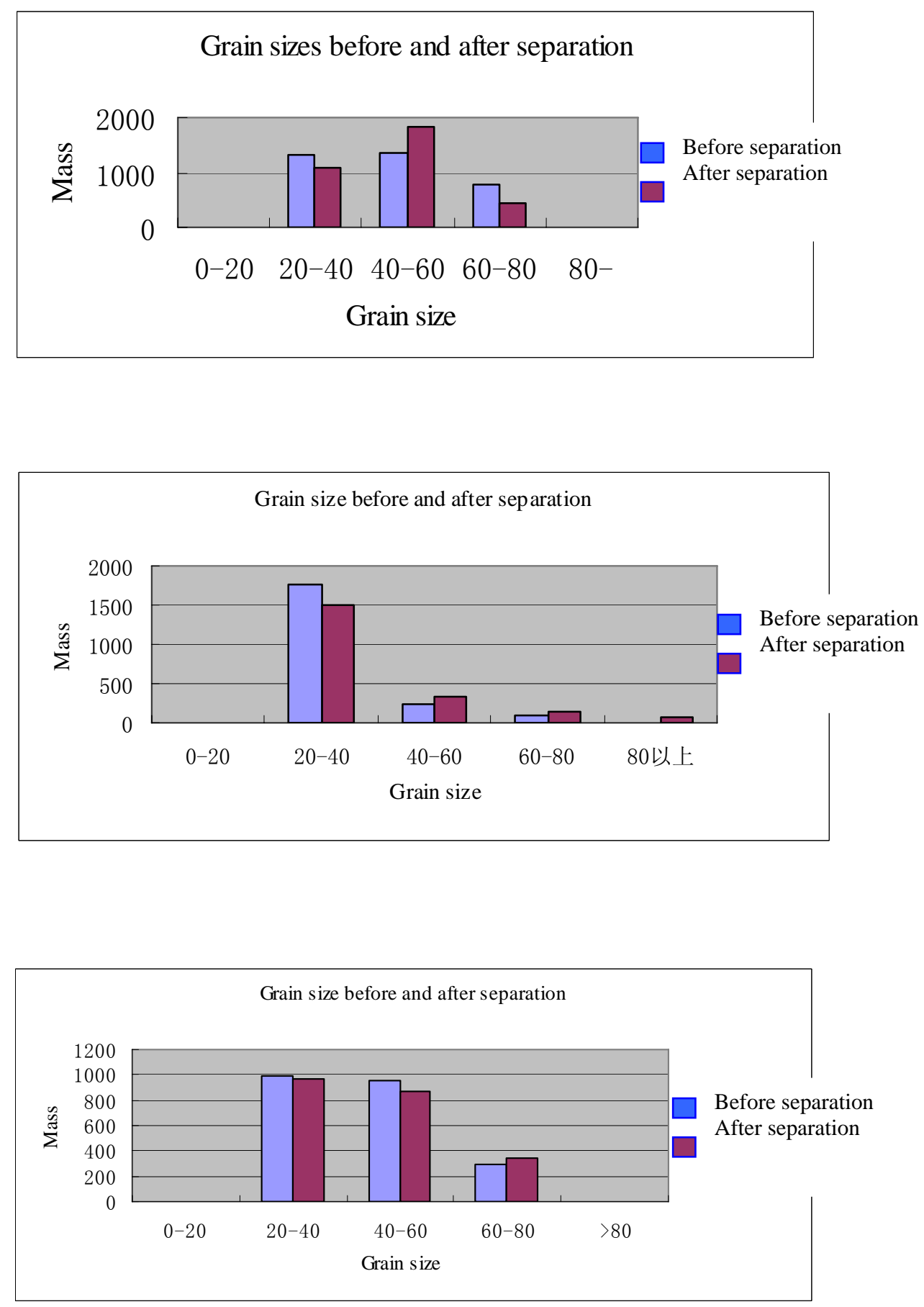

Fig. 7 Distribution of particle diameters

\section{Conclusions}

a. In case of highly viscous oil, the gravitational sedimentation of small-diameter sand particles be- comes more difficult.

b. Centrifugal separation is an effective way in the separation of small-diameter sand particles. 
c. For various oil viscosities, the separation efficiency of sand particles with diameters more than 100 eyes can reach 95 percent.

\section{Nomenclature}

\begin{tabular}{|c|c|}
\hline$g$ & acceleration due to gravity \\
\hline$\alpha$ & volume fraction \\
\hline$\rho$ & density \\
\hline$\vec{v}$ & velocity \\
\hline$\dot{m}_{p q}$ & $\begin{array}{l}\text { mass transfer from phase } \boldsymbol{p} \text { to } \\
\text { phase } \boldsymbol{q} \text {. }\end{array}$ \\
\hline $\begin{array}{l}\bar{\tau}_{q} \\
\tau_{q}\end{array}$ & shear stress \\
\hline$\vec{F}$ & body force \\
\hline $\bar{F}_{l i f t}$ & lift force \\
\hline$\vec{F}_{v m}$ & virtual mass force \\
\hline$K_{p q}$ & $\begin{array}{l}\text { interphase momentum } \\
\text { exchange coefficient }\end{array}$ \\
\hline$P$ & pressure \\
\hline$\vec{v}_{p q}$ & interphase velocity \\
\hline$\tau$ & particle relaxation time \\
\hline$d$ & particle diameter \\
\hline$n$ & number of phases \\
\hline$v_{r, s}$ & terminal velocity \\
\hline$e_{l s}$ & coefficient of restitution \\
\hline$C_{f r, l s}$ & $\begin{array}{l}\text { coefficient of friction } \\
\text { between } \boldsymbol{l} \text { and } s \text { solid phase }\end{array}$ \\
\hline$g_{0, l s}$ & $\begin{array}{ll}\text { radial distribution } \\
\text { coefficient }\end{array}$ \\
\hline
\end{tabular}

\section{Subscripts}

$$
\begin{array}{ll}
p & \text { - liquid phase } \\
q & \text { - liquid phase } \\
l & \text { - liquid phase } \\
s & \text { - solid phase }
\end{array}
$$

\section{References}

[1] J. M. Dalla Valle. Micromeritics. Pitman, London, 1948

[2] J. R. Richardson and W. N. Zaki. Sedimentation and fluidization: Part I [J]. Trans. Inst. Chem. Eng., 1954,32:35-53.

[3] M. Syamlal. The particle-particle drag term in a multiparticle model of fluidization. National Technical Information Service, Springfield, VA, 1987. DOE/MC/ 21353-2373, NTIS/DE87006500

[4] M. Syamlal and T. J. O'Brien. Computer simulation of bubbles in a fluidized bed [C]. AIChE Symp. Series, 1989, 85:22-31.

[5] J.P.Vandoormaal, G.D.Raithby. Enhancements of the SIMPLE method for predicting incompressible fluid flows. Heat Transfer[M], 1984(7), 147-163.

[6] J.L.Ferzieger, M.Peric. Computational Methods for Fluid Dynamics[M]. Heidelberg: Springer-Verlag, 1996.

[7] Guo jun zhang jun zheng-zhichu, The experimental development for a novel liquid-solid Separating system [C], Proceedings of the 7th National Congress on Hydrodynamics and 19th National Conference on Hydrodynamics 2005.

[8] Gong Dao-tong Wu Ying-xiang Zheng Zhi-chu,Numerical simulation of the separation of gravity settlement [C], Proceedings of the 7th National Congress on Hydrodynamics and 19th National Conference on Hydrodynamics 2005. 